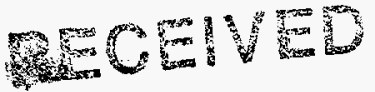 \\ JUN 051997 \\ OSTI \\ FINAL REPORT EDGE PLASMA AND CURRENT PROFILE DIAGNOSTIC DEVELOPMENT
}

\author{
J.M. MCCHESNEY
}

\author{
Prepared under \\ Grant No. DE-FG03-92ER54150 \\ for the U.S. Department of Energy
}

\section{DISCLAIMER}

\begin{abstract}
This report was prepared as an account of work sponsored by an agency of the United States Government. Neither the United States Government nor any agency thereof, nor any of their employees, makes any warranty, express or implied, or assumes any legal liability or responsibility for the accuracy, completeness, or usefulness of any information, apparatus, product, or process disclosed, or represents that its use would not infringe privately owned rights. Reference herein to any specific commercial product, process, or service by trade name, trademark, manufacturer, or otherwise does not necessarily constitute or imply its endorsement, recommendation, or favoring by the United States Government or any agency thereof. The views and opinions of authors expressed herein do not necessarily state or reflect those of the United States Government or any agency thereof.
\end{abstract}




\section{DISCLAMMER}

Portions of this document may be illegible in electronic image products. Images are produced from the best available original document. 


\section{DISCLAIMER}

This report was prepared as an account of work sponsored by an agency of the United States Government. Neither the United States Government nor any agency thereof, nor any of their employees, makes any warranty, express or implied. or assumes any legal liability or responsibility for the accuracy, completeness, or usefulness of any information, apparatus, product, or process disclosed, or represents that its use would not infringe privately owned rights. Reference herein to any specific commercial product, process. or service by trade name, trademark, manufacturer, or otherwise, does not necessarily constitute or imply its endorsement, recommendation, or favoring by the United States Government or any agency thereof. The views and opinions of authors expressed herein do not necessarily state or reflect those of the United States Government or any agency thereof. 
GA-C22322

\title{
FINAL REPORT \\ EDGE PLASMA AND CURRENT PROFILE DIAGNOSTIC DEVELOPMENT
}

\author{
J.M. MCCHESNEY \\ Prepared under \\ Grant No. DE-FG03-92ER54150 \\ for the U.S. Department of Energy
}

GA PROJECT 3937

MAY 1997 


\section{CONTENTS}

OVERVIEW

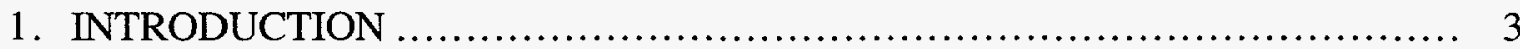

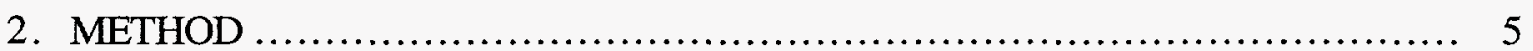

3. DEVELOPMENT OF DIAGNOSTIC HARDWARE AND

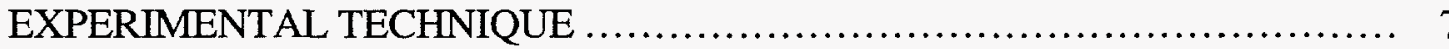

3.1. Background ............................................................. 7

3.2. Neutral Beam System .......................................... 7

3.3. Optical System and Fluorescence Detection Development.................. 9

3.4. Data Acquisition ................................................. 12

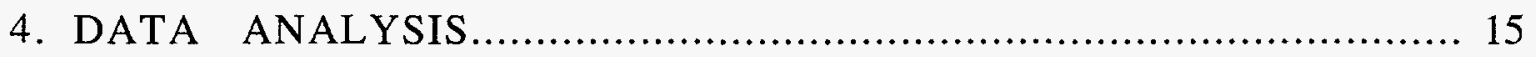

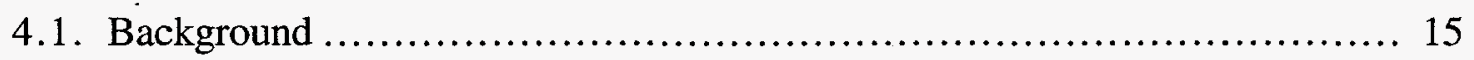

4.2. Beam-Plasma Model ................................................. 16

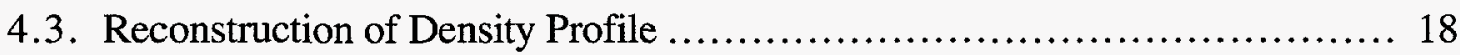

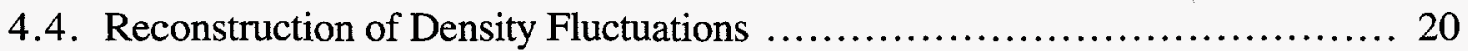

4.5. Numerical Example ................................................. 22

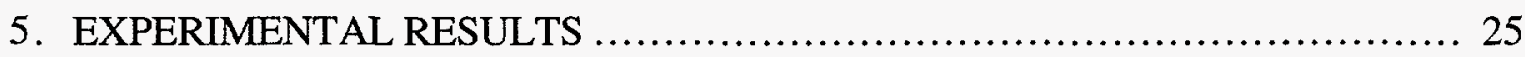

6. SUMMARY AND FUTURE DIRECTIONS .................................. 29

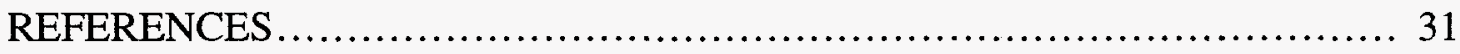

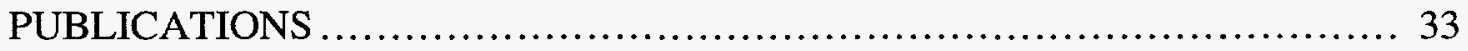




\section{FIGURES}

1. $\operatorname{Li} 2 \mathrm{~s} 2 \mathrm{~S}, \mathrm{Li} 2 p 2 \mathrm{P}$ and, $\mathrm{Li}(\mathrm{n}=3$ ) level populations, normalized to the initial beam intensity, calculated for a $25 \mathrm{keV}$ beam traversing a plasma with a size and density representative of the TEXT tokamak

2. Schematic showing the TEXT-U lithium beam system $\ldots \ldots \ldots \ldots \ldots \ldots \ldots \ldots \ldots . \ldots$

3. Oscilloscope trace showing a stable $30 \mathrm{keV}$ ion beam on the test stand ............ 9

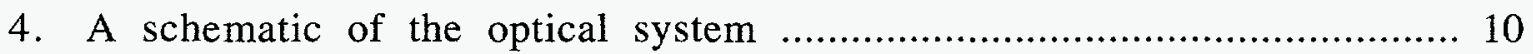

5. Raw data from a single spatial channel demonstrating the background subtraction signal

6. Schematic showing a single channel of the data acquisition system for the TEXT lithium beam

7. (a) Sample calculation of the $2 p$ level population as a function of beam penetration. (b) Relative BES RMS fluctuation level as a function of wavenumber with $\left(\tilde{n}_{\mathrm{e}} / \mathrm{n}_{\mathrm{e}}\right)_{\mathrm{RMS}}=0.2$

8. Numerical example demonstrating unfolding technique

9. (a) Example of lithium BES data (solid points) obtained during TEXT discharge 173987. (b) The unfolded electron density profile obtained from the BES profile showin in (a) (solid points) and the density profile obtained using Eq. (9) (solid line)

10. The time series of reconstructed electron density profiles obtained during TEXT discharge 189096 


\section{OVERVIEW}

This is the final report covering the research conducted under DOE Grant No. DEFG03-92ER54150 entitled "Edge Plasma and Current Profile Diagnostic Development." It is intended to summarize the investigation and will go into somewhat more detail regarding the aims, techniques, and results of the project research than the standard technical progress reports submitted previously. During the course of this work we developed and implemented an atomic beam-based diagnostic technique for investigating edge plasma density behavior on the TEXT Tokamak. The project required the modification of the existing $100 \mathrm{keV}$ TEXT lithium beam to operate at $20-30 \mathrm{keV}$ and the addition of a new 20 detector chain to collect the fluorescence emissions. The modifications were completed and experimental density profiles were unfolded using a new inversion technique. 


\section{INTRODUCTION}

The pivotal role played by edge physics in tokamak transport and performance is widely appreciated but not completely understood at this time. The evident bifurcation in transport behavior during the $\mathrm{L}-$ to $\mathrm{H}$ - transition and the modification of the edge region by ELMs are just two examples of this relationship [1,2]. There is a clear need to understand the role that turbulence plays in the plasma transport problem. Various workers have seen a suppression of both the magnetic and plasma density fluctuations during the transition. It is clear that the turbulence and transport behavior are linked and we are coming to an understanding of the transition evolution, based on a model of shear suppression of the turbulence and the creation of a transport barrier [3,4].

Atom and ion beams of many different types have been used as diagnostics of fusion plasma parameters. One subset of these beam diagnostics is that for which the measured quantity is light emitted by beam particles. High power heating beams and low power diagnostic beams have both been used, and the general technique is called beam emission spectroscopy. Beams of neutral lithium atoms are a common choice since they are relatively easy to produce, lithium is a benign plasma contaminant, and the lithium resonance transition line lies in the visible part of the spectrum. Fast neutral lithium beams have been used as probes of plasma edge densities in ASDEX [5], TEXTOR [6], and CHS [7], of local magnetic field angle in TEXT [8] and of plasma density fluctuations in DIII-D [9]. Neutral lithium beams have also been proposed as targets for charge-exchange recombination spectroscopy of plasma impurity ions [10]. 


\section{METHOD}

The collisional excitation of a small $(\sim 2.5 \mathrm{~cm}$ diameter) beam of neutral lithium atoms has been used to measure the plasma density profile in the edge region of the TEXT tokamak. The beam undergoes exciting and ionizing collisions with plasma particles as it transits the edge region. The resulting fluorescence from the excited $2 \mathrm{P}$ state may be collected using visible light optics and analysis of the fluorescence behavior allows for a determination of the underlying plasma density behavior in the observation region. Observing the fluorescence emitted transverse to the beam yields the good spatial resolution while the relatively large atomic cross-sections for collisional excitation yield a large fluorescence rate which in turn translates into good temporal resolution. Because the measurement is made continuously throughout the shot it is particularly well suited to studying transient effects (such as the L-H transition and ELMs) which may be crucial to the underlying transport. Since the light is emitted along the entire path of the beam, multichannel methods are easily applied to address the issues of profile evolution. Precise knowledge of the viewpoint locations is obtained through prior in-situ spatial calibration.

A neutral lithium beam was injected radially into the plasma on the tokamak midplane, and was viewed from below. The neutral beam intensity was $1-2 \mathrm{~mA}$ at a beam energy of $20-30 \mathrm{keV}$, and the diameter of the beam in the plasma was $2.5 \mathrm{~cm}$. When the lithium beam entered the plasma it was predominantly in the ground $L i 2 s 2 \mathrm{~S}$ state. Collisions between the beam and plasma electrons and ions populated higher energy levels in the beam atoms and attenuated the beam due to ionizing collisions. This is illustrated in Fig. 1, which shows the populations of the $L i 2 s 2 \mathrm{~S}, L i 2 p 2 p$, and $L i(n=3)$ levels calculated for a TEXT-like plasma. This calculation solves a set of coupled differential equations for the various $L i$ states, using a database of measured or calculated cross sections for collisional and radiative transitions among the various states [11]. The intensity light from the $L i I(2 s 2 S-2 p 2 P$ ) resonance transition is directly proportional to the population of atoms in the $2 p$ state and is the measured quantity. The useful diagnostic range of the beam in the plasma is on the order of $20 \mathrm{~cm}$, limited by beam attenuation and by saturation of the ratio of the populations of atoms in the $2 \mathrm{~S}$ and $2 p$ states. The $L i 2 p$ level population and the $670.8 \mathrm{~nm}$ emission intensity are not directly proportional to the local plasma density, due to attenuation of the beam and the finite lifetime of the $2 p$ level, so the electron-density profile must be "unfolded" from the fluorescence profile. The unfolding process consists of finding a putative electron-density profile for which the $L i 2 p$ population profile matches the measured $670.8 \mathrm{~nm}$ emission 
profile. Because of the large amount of data to be analyzed (up to 50,000 profiles per shot), a new faster technique was developed to do this calculation (see Section 4).

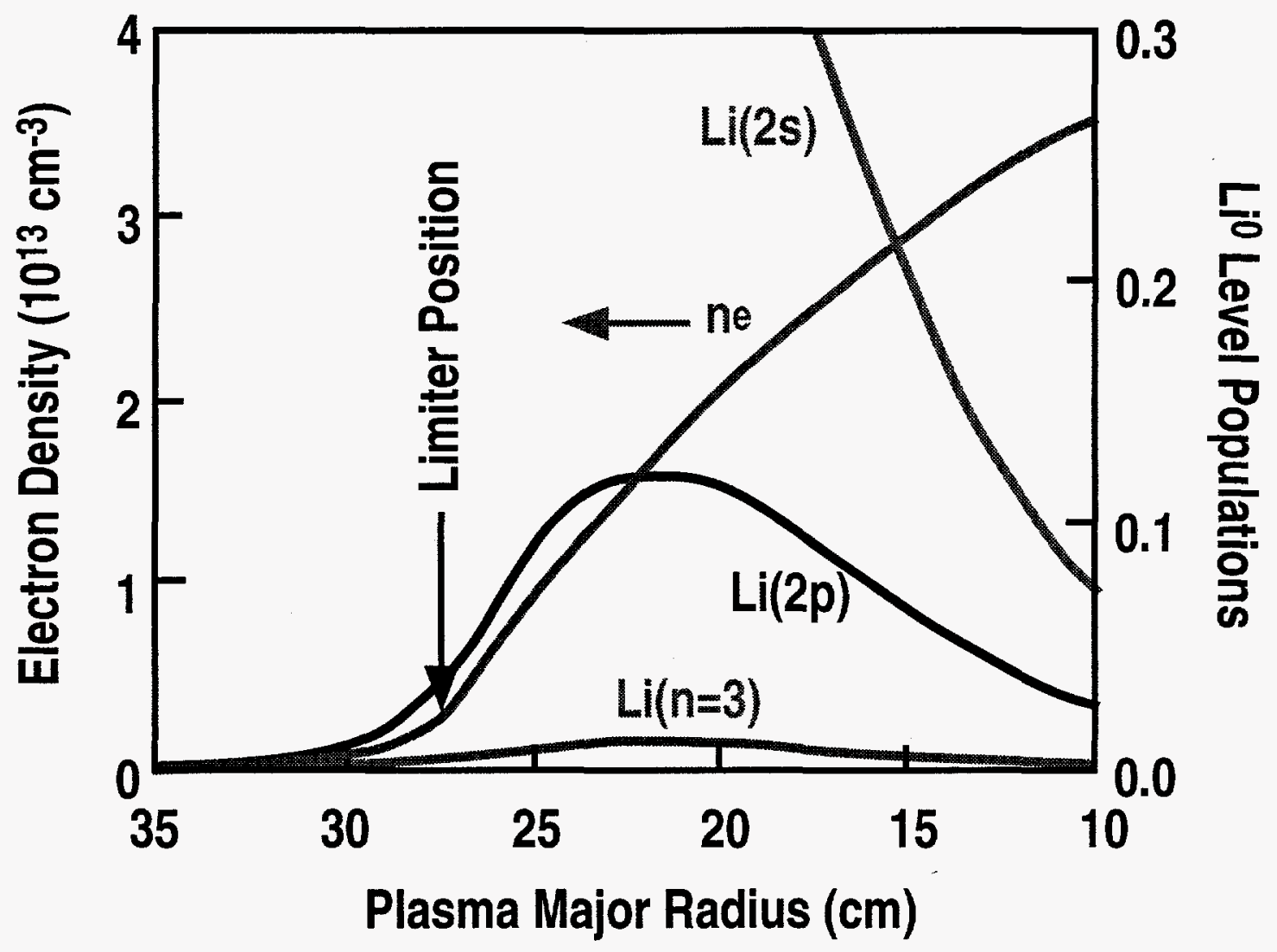

Fig. 1. $\mathrm{Li} 2 \mathrm{~s} 2 \mathrm{~S}$, $\mathrm{Li} 2 p 2 \mathrm{P}$ and, $\mathrm{Li}(n=3)$ level populations, normalized to the initial beam intensity, calculated for a $25 \mathrm{keV}$ beam traversing a plasma with a size and density representative of the TEXT tokamak. 


\section{DEVELOPMENT OF DIAGNOSTIC HARDWARE AND EXPERIMENTAL TECHNIQUE}

\subsection{BACKGROUND}

The development of a diagnostic system capable of performing the above measurements on the TEXT tokamak at the University of Texas at Austin has involved work in a number of areas. Work on this project may be naturally organized according to the following three subareas, (a) neutral beam system, (b) optical system, and (c) data acquisition. Results in each of these areas are summarized below.

\subsection{NEUTRAL BEAM SYSTEM}

A schematic diagram of the experimental apparatus is shown in Fig. 2. The lithium beam source and accelerator are a modified version of the higher energy accelerator used earlier on TEXT to measure the plasma current distribution [8]. The selected beam energy of $25 \mathrm{keV}$ was a compromise between penetration and spatial resolution. The beam penetration into the plasma increases with beam energy. On the other hand, the fluorescence is smeared out in space by a distance on the order of the beam velocity times the lifetime of the $2 \mathrm{P}$ excited state and higher energy beams have poorer spatial resolution. For typical plasma conditions on TEXT, the beam was attenuated to $20 \%$ of its original intensity in about $20 \mathrm{~cm}$, and the fluorescence decay length due to radiative decay was $2.4 \mathrm{~cm}$.

The ion source provided a dense, monoenergetic, focused beam of lithium ions for subsequent neutralization and injection into the tokamak periphery. Based on our earlier work [8] we chose a source based on the thermoemissive glass beta-eucryptite [12-14]. This style of source was far easier to use and much more reliable than a discharge-style ion sources. A supply of the material was made from the constituent materials alumina, silica, and lithium carbonate. Because of the emission limit of about $1 \mathrm{~mA} / \mathrm{cm}^{2}$ for this material, a large area source followed by ion beam compression was employed. An emitter diameter of $2.5 \mathrm{~cm}$ was chosen as a good compromise between desired current, achievable beam compression, and the available vacuum envelope for the accelerator components. The sources were prepared by melting successive coats of glass onto a suitable substrate disc of sintered tungsten [15] mounted in a vacuum coating stand constructed for this purpose. These source discs could then be easily switched in and out 
of the ion source when depleted. The ion emission level was easily regulated by controlled heating of the source.

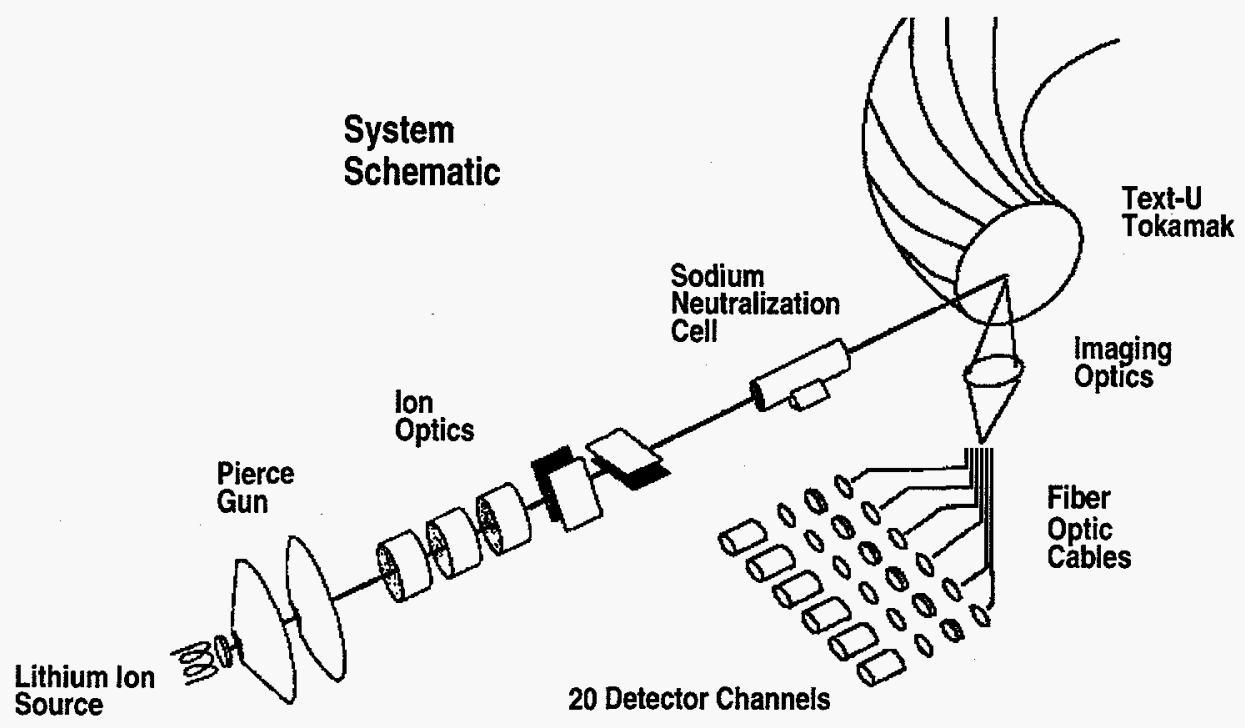

Fig. 2. Schematic showing the TEXT-U lithium beam system. This system operated between $5-30 \mathrm{keV}$ and could provide neutral currents of about $2 \mathrm{~mA}$.

Compression of the emitted ions into a roughly $1 \mathrm{~cm}$ diameter beam was achieved using a spherically focusing Pierce geometry [16,17]; the specific design was determined from space-charge calculations. The compact design allowed for ion extraction at full energy, simple control of source heating, and simple (low frequency) modulation of the ion beam. The operating energy of $30 \mathrm{keV}$ was chosen as a compromise between good plasma penetration and spatial smearing of the experimental resolution due to finite lifetime effects. Vacuum pumping of the entire accelerator was provided by two $500 \mathrm{\ell} / \mathrm{s}$ turbomolecular pumps. Because of the relatively high perveance of the beam an electrostatic Einzel lens was used as a relay lens to image the beam into the neutralizer. A suppresser ring and separate power supply were used to prevent electron back streaming from the beam line back up the accelerator to the source disc. Test stand measurements of the ion beam allowed us to identify the proper operating points for the formation and transmission of stable beams of several milliamperes (Fig. 3). 


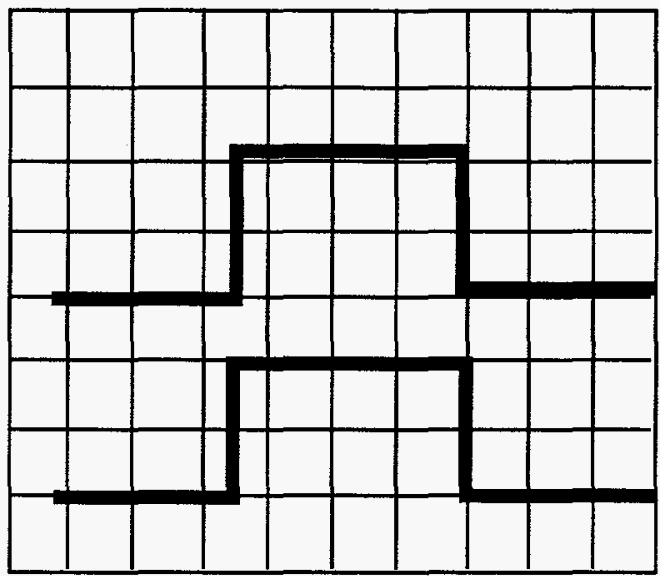

Fig. 3. Oscilloscope trace showing a stable $30 \mathrm{keV}$ ion beam on the test stand. The upper trace is the extracted ion current ( $2 \mathrm{~mA} / \mathrm{div})$ and the lower trace is the transmitted ion current to a $2.5 \mathrm{~cm}$ diameter faraday cup ( $1 \mathrm{~mA} / \mathrm{div})$.

The neutralization of $\mathrm{Li}^{+}$ions to $\mathrm{Li}$ neutrals was performed in a sodium vapor, since at the beam energies of interest neutralization efficiencies of $80 \%-95 \%$ are possible. Because of the high perveance and rapid radial blowup of the ion beam the neutralization was done over a very short path length and as close as possible to the source to minimize beam spread. At the same time, care was taken to prevent excessive coating of the source with metallic sodium. A shortened "heat-pipe" style sodium vapor cell [18] having an active length $5 \mathrm{~cm}$, a bore diameter of $2.3 \mathrm{~cm}$, and an overall length of $15 \mathrm{~cm}$ was used. The optimum temperature range was found to be $260^{\circ} \mathrm{C}-275^{\circ} \mathrm{C}$.

\subsection{OPTICAL SYSTEM AND FLUORESCENCE DETECTION DEVELOPMENT}

The arrangement of the optics used to measure the $670.8 \mathrm{~nm}$ Lil emission is shown in Fig. 4. It was designed to have a high photon collection efficiency, uniform spatial sensitivity (within each channel), and to discriminate between lithium light and plasma background light. The pair of achromatic lenses L1 and L2 focused the light emitted from the lithium beam onto an optical fiber assembly. Because of the limited numerical aperture of the fibers, the optical axis was centered on the portion of the beam being measured. The lenses were located at the same toroidal position as the beam, but there was no port at this major radius. A straight, angled optical axis was not possible because of limited space. For this reason, an optical wedge is used to bend the optical axis by 8 degrees. The lens $\mathrm{L} 1 \mathrm{had}$ a diameter of $75 \mathrm{~mm}$, a focal length of $500 \mathrm{~mm}$, and subtended a solid angle of $1.8 \times 10^{-2} \mathrm{sr}$. Lens $\mathrm{L} 2$ has a $200 \mathrm{~mm}$ focal length. Both were used at infinite conjugate ratio, for an effective f-number of approximately 2 . A single 
simple lens with the same diameter and focal length would have significant spherical aberration which would have produced cross-talk between adjacent channels. All lenses were anti-reflection coated to maximize the signal strength at the detectors.

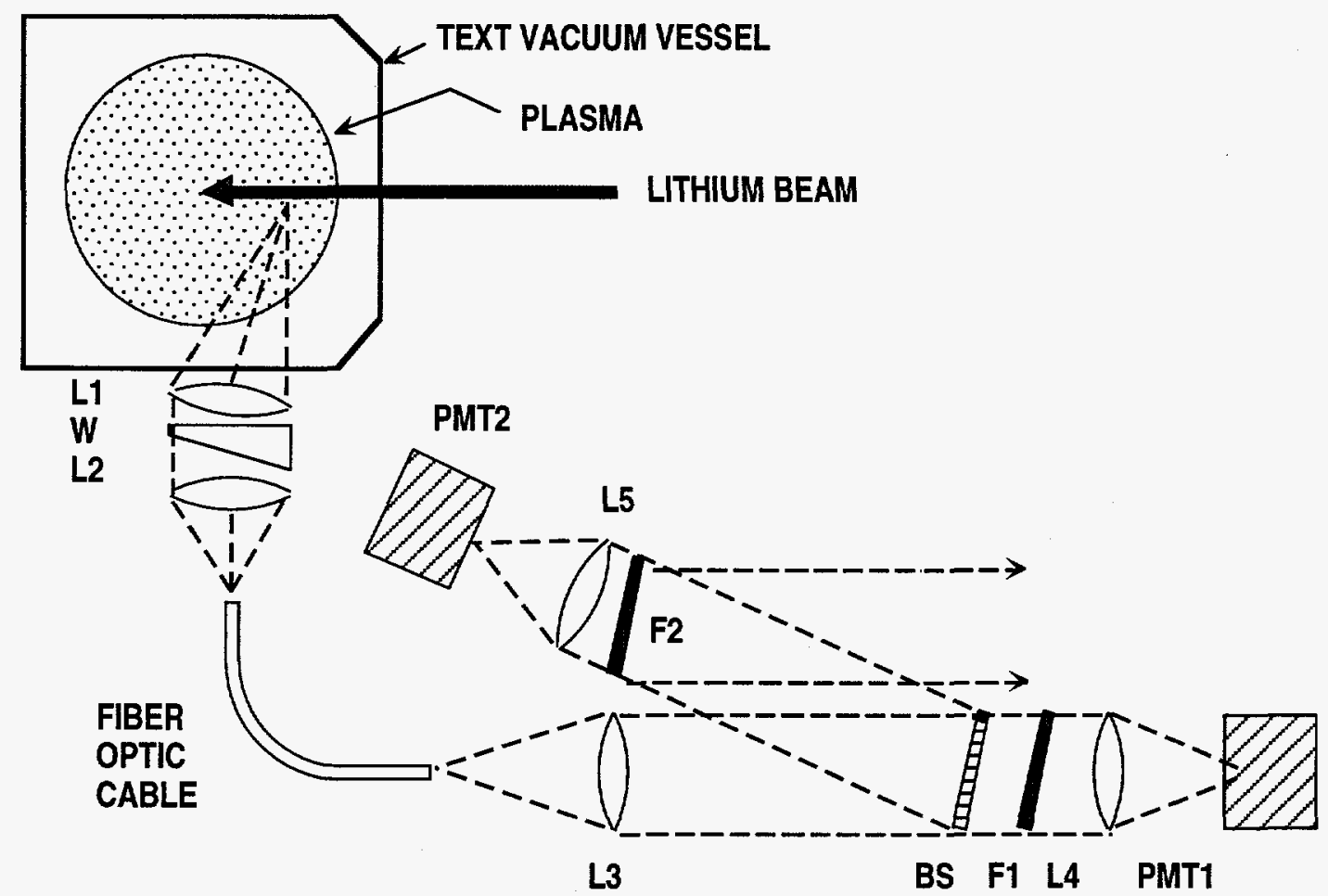

Fig. 4. A schematic of the optical system. Lens $L 1$, wedge $W$, and lens $L 2$ focus light from the lithium beam onto an array of 20 fiber-optic bundles. The remainder of the figure shows the optics for one of the ten spatial channels. Light from the fiber bundle is collimated by lens L3. Interference filter F1 passes light at $670.8 \mathrm{mn}$ (bandwidth $=1.0 \mathrm{~nm}$ ) and reflects light at other wavelengths. Light that gets through $F 1$ is focused by lens L4 onto photomultiplier PMT1. This light has two components: Li (2S-2P) signal and plasma background. Light reflected from $F 1$ goes to $F 2$, an interference filter centered at $685 \mathrm{~nm}$ (bandwidth $=3.0 \mathrm{~nm}$ ). Light that gets through $\mathrm{F} 2$ is focused by lens L5 onto photomultiplier PMT2. This light has only a plasma background component.

The fiber optic assembly consisted of 20 fiber cables, each of which consisted of several thousand individual fibers. The cables had rectangular ends, $3 \mathrm{~mm}$ by $12 \mathrm{~mm}$. At the tokamak, the 20 cable ends were butted together to form an overall $60 \mathrm{~mm}$ by $12 \mathrm{~mm}$ rectangle. The magnification is approximately 2.5 , so each channel covered approximately $7.5 \mathrm{~mm}$, and the total length of beam observed in the plasma was approximately $150 \mathrm{~mm}$. The remainder of this description is for one of twenty identical channels: Lens F3 had a diameter of $50 \mathrm{~mm}$ and focal length of $100 \mathrm{~mm}$, and collimates the light emitted from the opposite end of the fiber cable. The collimated light was then 
split into two parts of approximately equal intensity by a beamsplitter ("BS" in the figure). One part was focused, after passing through interference filter $F 1$, onto the detector PMT1. The pass band of filter F1 had a central wavelength centered on the (Doppler shifted) $670.8 \mathrm{~nm}$ lithium line, and a FWHM of $1.0 \mathrm{~nm}$. Because of the finite solid angle subtended by lens L1, the $670.8 \mathrm{~nm}$ lithium line had a Doppler broadened width of $0.3 \mathrm{~nm}$ at the filter. Lens L4 (and L5) was a $50 \mathrm{~mm}$ diameter, $50 \mathrm{~mm}$ focal length aspheric lens. It produced a 1.5 by $3 \mathrm{~mm}$ image of the 3 by $6 \mathrm{~mm}$ rectangular fiber bundle end on the photocathode of PMT1 (Hamamatsu R636) Ga-As photomultiplier with approximately $10 \%$ quantum efficiency at this wavelength. Each of the 20 detectors therefore measured the integral of the LiI emission profile over a $7.5 \mathrm{~mm}$ length of the beam path. But according to the manufacturer, the R636 photomultiplier tubes used for this experiment had a non-uniform sensitivity over their active area. This sensitivity can vary by a factor of 2 over the $1.5 \mathrm{~mm}$ width of the image [24], the distance corresponding to the $7.5 \mathrm{~mm}$ length of the beam in the plasma viewed by the channel. This could have introduced errors in the experimental determinations of the integrals over the $7.5 \mathrm{~mm}$ lengths of the spatial channels, since the intensity could vary significantly in this distance. If, for example, the brightest part of a channel were imaged onto the least sensitive part of a detector, that light would have been underrepresented in the integral and the signal from that channel would have been systematically too low. To circumvent this problem, the fiber bundles were assembled with randomized individual fibers. This way, any part of the image of a particular channel was distributed over the entire active area of the photomultiplier, and all sections of the image were detected with uniform average sensitivity.

Plasma continuum light near $670.8 \mathrm{~nm}$ was also transmitted through interference filter F1, so that light measured by this photomultiplier was the sum of two parts, a "signal" component from the lithium beam and a "background" component from the plasma continuum, notably molecular hydrogen light from the plasma edge. As shown in Fig. 5, the signal to background ratio was approximately 4:1. For slow time scales and reproducible plasmas, the background measured on PMT1 with the lithium beam off can be subtracted from the "signal plus background" measured on PMT1 with the lithium beam on. For faster time scales or transient events, however, the background intensity was not be reproducible from shot to shot, and its subtraction introduced significant errors. This is the reason for the second photomultiplier PMT2, which was designed to measure the plasma background light simultaneously with the signal plus background, at a slightly different wavelength. 


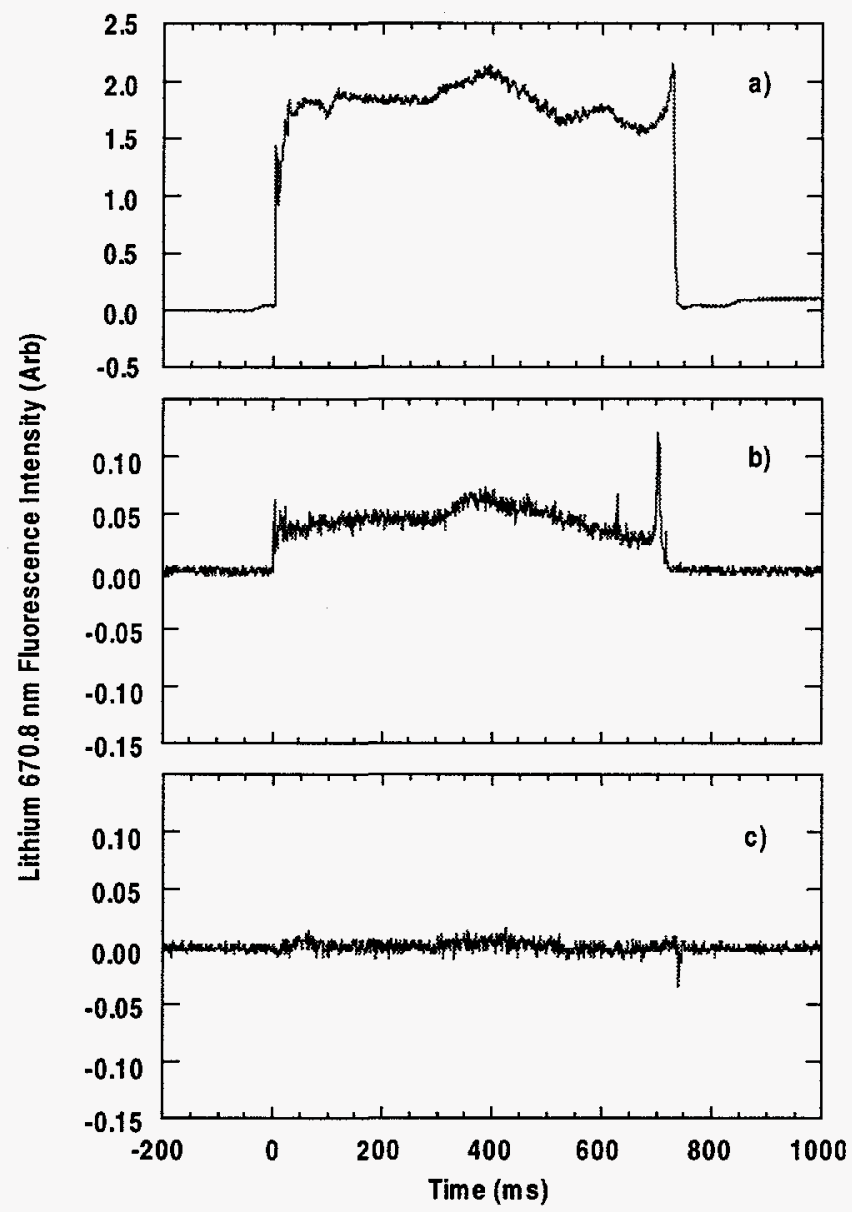

Fig. 5. Raw data from a single spatial channel demonstrating the background subtraction signal. (a) Shot 223443, beam on, signal + background (PMT1PMT2), (b) shot 223434, beam off, background only (PMT1 only), (c) shot 223442, beam off, background only (PMT1 - PMT2).

\subsection{DATA ACQUISITION}

Filter F2 had a central wavelength of $685 \mathrm{~nm}$ and a FWHM of $3 \mathrm{~nm}$. As shown in Fig. 6, the signals from both PMT1 and PMT2 signal currents were converted to voltages by Analog Modules, Inc. model 341-4 amplifiers, and filtered by TTE, Inc. 4, 40, or $400 \mathrm{kHz}$ anti-aliasing filters. The signals are then fed to the differential inputs of a DSP, Inc model 2860 digitizer. The signal from PMT2 was attenuated by an amount adjusted to give zero digitized difference when there is plasma but no lithium beam. This adjustment is illustrated in Fig. 5, which shows data from one of the 20 channels for the following cases; (a) signal plus background (PMT1+PMT2), (b) background only (PMT1 only), (c) a shot with the Li beam off, showing good background subtraction (PMT1-PMT2) 


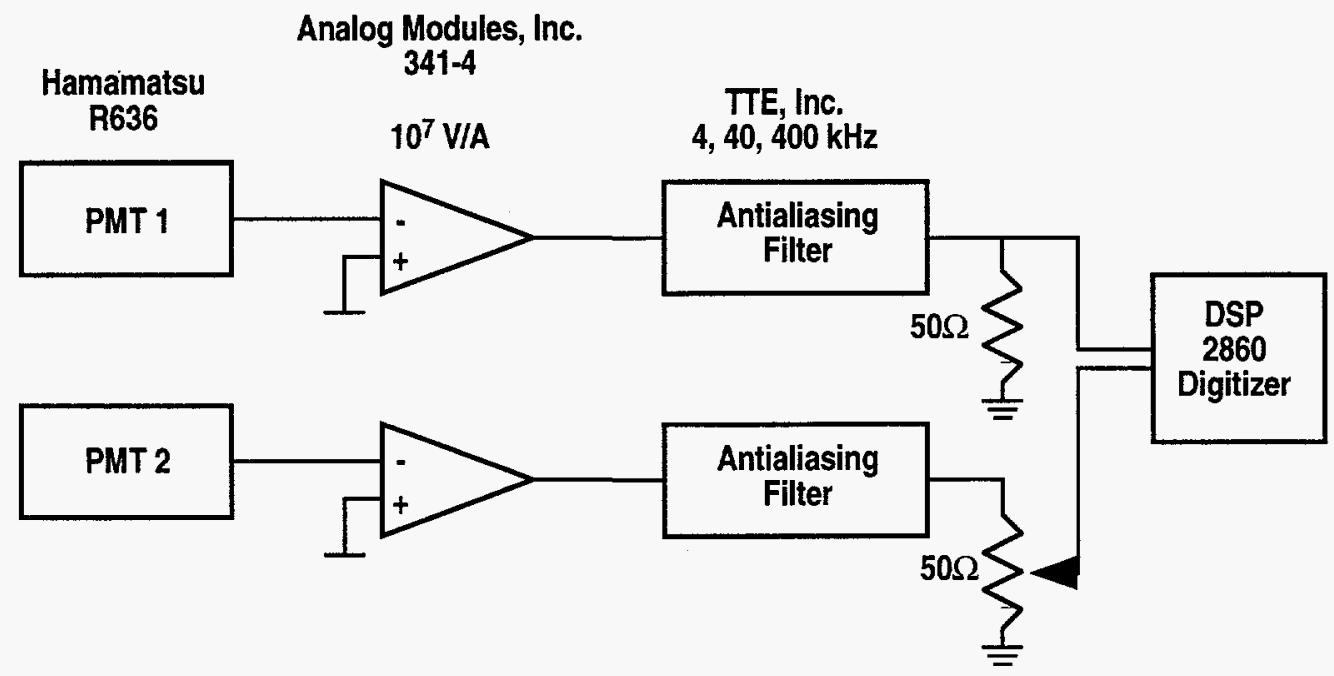

Fig. 6. Schematic showing a single channel of the data acquisition system for the TEXT lithium beam. 


\section{DATA ANALYSIS}

\subsection{BACKGROUND}

The inversion of the fluorescence light to obtain the underlying electron density profile is evidently not a trivial problem. Many competing atomic processes occur simultaneously and the local fluorescence signal is not simply proportional to the local electron density. We worked with an existing computer code, LIBEAM developed at UT [19] and modified at GA for the TEXT program. LIBEAM is a forward code; i.e., it uses a set of rate equations for calculating a fluorescence emission profile given density and temperature profiles in the plasma edge. It is based on a ten state collisional-radiative beam-plasma model capable of accurately predicting the beam penetration and fluorescence emission profile for lithium beam energies between $5 \mathrm{keV}$ and a few $\mathrm{MeV}$, given input density and temperature profiles. The code has been calibrated with experimental light profiles obtained from the TEXT lithium beam. Very good agreement was found between the code prediction and the observed fluorescence profiles for plasma densities up to $7 \times 10^{13} \mathrm{~cm}^{-3}$ [20].

Several methods have been proposed to unfold the underlying electron density from the observed fluorescence profile [21-23]. These methods will not be discussed in any detail here, but they require the integration of the various rate equations describing the propagation of a lithium beam through a plasma and are prone to various numerical instabilities. In addition, they are time consuming and are therefore not applicable to the unfolding of density fluctuations. For instance, in order to analyze fluctuation data at $500 \mathrm{kHz}$, it is necessary to digitize at least $1 \mathrm{MHz}$. Each plasma discharge may therefore generate several times $10^{6}$ profiles. It is often desirable that the analysis be performed in between plasma discharges, i.e., within a few minutes. The present work demonstrates a novel method for unfolding the electron density profile from the lithium beam fluorescence and also shows how the underlying electron density fluctuations can be obtained from the fluorescence light fluctuations. We are still not to the point where the codes are straightforward to use to obtain the fluctuating density values. Our studies indicate that, because of saturation effects, the intensity fluctuations observed become smaller and smaller at higher densities (for a given $\tilde{n} / n$ ). This tends to obscure density fluctuations level determinations at the highest densities, although the intensity fluctuations are still an indicator of the underlying turbulence spectrum. 


\subsection{BEAM-PLASMA MODEL}

The beam fluorescence observed at a particular point in the plasma is not only a function of the local plasma parameters but also depends on excitation and ionization that occurs upstream from the point under consideration. In order to account for these effects a collisional-radiative model has been developed at General Atomics which calculates the populations of the various energy levels as the beam propagates through the plasma. This 10 state model includes lithium states up to the principal quantum number $n=5$ and includes $\mathrm{C}^{+6}$ as the typical impurity ion. The actual levels used are as follows: $n=1 \rightarrow$ $1 s, n=2 \rightarrow 2 s, n=3 \rightarrow 2 p \pi, n=4 \rightarrow 2 p \sigma, n=5 \rightarrow 3 s, n=6 \rightarrow 3 p d, n=7 \rightarrow 4 s, n=$ $8 \rightarrow 4 p d f, n=9 \rightarrow 5$ spdf and $n=10 \rightarrow$ ionization continuum. The cross-sections were obtained from a wide variety of sources, both experimental and theoretical.

We assume that a beam of velocity $v_{0}$ is propagating through a hydrogen plasma containing only $\mathrm{C}^{+6}$ as an impurity. The $\mathrm{C}^{+6}$ is assumed to be distributed throughout the plasma in such a manner that $Z_{\text {eff }}$ is constant. The rate equations governing the transmission of the atomic beam through a plasma are as follows,

$$
\begin{aligned}
v_{o} \frac{d P_{n}}{d x}= & -k_{n}^{e(i)} n_{e} P_{n}+\sum_{m<n}\left\{k_{m n}^{e(e)} n_{e} P_{m}-\left[k_{n m}^{e(d)} n_{e}+A_{n m}\right] P_{n}\right\} \\
& -\sum_{m<n}\left\{k_{m n}^{e(e)} n_{e} P_{n}-\left[k_{m n}^{e(d)} n_{e}+A_{n m}\right] P_{m}\right\} \\
& -v_{o} \sigma_{n}^{i(i)} n_{i} P_{n}+v_{o} n_{i} \sum_{m<n}\left[\sigma_{m n}^{i(e)} P_{m}-\sigma_{m n}^{i(d)} P_{n}\right] \\
& v_{o} n_{i} \sum_{m>n}\left[\sigma_{m n}^{i(e)} P_{n}-\sigma_{m n}^{i(d)} P_{m}\right]
\end{aligned}
$$

where

$$
\begin{aligned}
& v_{0}=\text { beam velocity, } \\
& P_{n}=\text { density of level } n, \\
& k_{n}^{e(i)}=\text { ionization rate due to electron impact, } \\
& k_{m n}^{e(e)}=\text { electron excitation rate }(m \rightarrow n), \\
& k_{m n}^{e(d)}=\text { electron de-excitation rate }(m \rightarrow n), \\
& \quad=k_{m n}^{e(e)} g m / g n, \text { for } T_{e}>\Delta E_{m n},
\end{aligned}
$$

$A_{m n}=$ rate of spontaneous de-excitation (Einstein coefficient), 
$\sigma_{n}^{i(i)}=$ effective ion loss cross-section, due to impact ionization and charge exchange, $\sigma_{m n}^{i(e)}=$ effective cross-section for ion impact excitation $(m \rightarrow n)$, $\sigma_{m n}^{i(d)}=$ effective cross-section for ion impact de-excitation $(m \rightarrow n)$.

The hydrogen and impurity ion impact cross-sections are lumped together for compactness. In typical fusion plasmas $v_{\mathrm{i}} \ll<v_{0}$ so that ion excitation and ionization processes assume the form $n_{\mathrm{i}} \sigma v_{0}$. For the electrons however, $v_{0} \ll<v_{\mathrm{e}}$ and the electron rate coefficients $k^{\mathrm{e}}$ must be obtained by averaging the respective cross-sections over a Maxwellian velocity distribution (i.e., $\left.k_{m n}^{e(e)}=\left\langle\sigma_{m n}^{e(e)} v_{e}\right\rangle\right)$.

Figure 7(a) shows the lithium $2 p$ population (solid line) calculated using the electron density profile shown (dashed line). The $2 p$ population is directly proportional to the expected light intensity observed at the detectors. A $25 \mathrm{kV}$ lithium beam propagates into a model plasma slab of density $\bar{n}_{e}=2 \times 10^{13} \mathrm{~cm}^{-3}$ and temperature $1 \mathrm{keV}$. Superimposed on the $\bar{n}_{e}$ is a fluctuating component of the density with wavenumber equal to $1.0 \mathrm{~cm}^{-1}$ and RMS amplitude equal to $0.2 \bar{n}_{e}$. It is clear from this figure that the strong plasma electron density fluctuations are smeared out in the BES signal. This effect is caused by the relatively long radiative lifetime of the $2 p$ excited state ( $27 \mathrm{~ns}$ ) and also by the much higher cross-sections for ionization from the higher states compared with the ground state. By varying the wavenumber it is possible to plot $\left(\tilde{n}_{2 p} / n_{2 p}\right)_{\mathrm{RMS}}$ as a function of wavenumber $k$, see Fig. 7(b). The relative light fluctuation levels which are directly proportional to $\left(\tilde{n}_{2 p} / n_{2 p}\right)_{\mathrm{RMS}}$ are a strong function of $\mathrm{k}$. Clearly for large $k,\left(k>3 \mathrm{~cm}^{-1}\right)$ the fluctuations are essentially washed out by the finite lifetime effects of the $2 p$ state. For $\left(k<3 \mathrm{~cm}^{-1}\right.$ ) this effect is reduced but is still very significant. It was found that $\left(\tilde{n}_{2 p} / n_{2 p}\right)_{\mathrm{RMS}}$ was not a very strong function of $\left(\tilde{n}_{e} / n_{e}\right)_{\mathrm{RMS}}$ or of $\bar{n}_{e}$. Since it is desirable that relative density fluctuations of the order of $5 \%-10 \%$ be measured, all noise sources must be reduced to about one-tenth of this value, i.e., about $0.5 \%-1.0 \%$. 

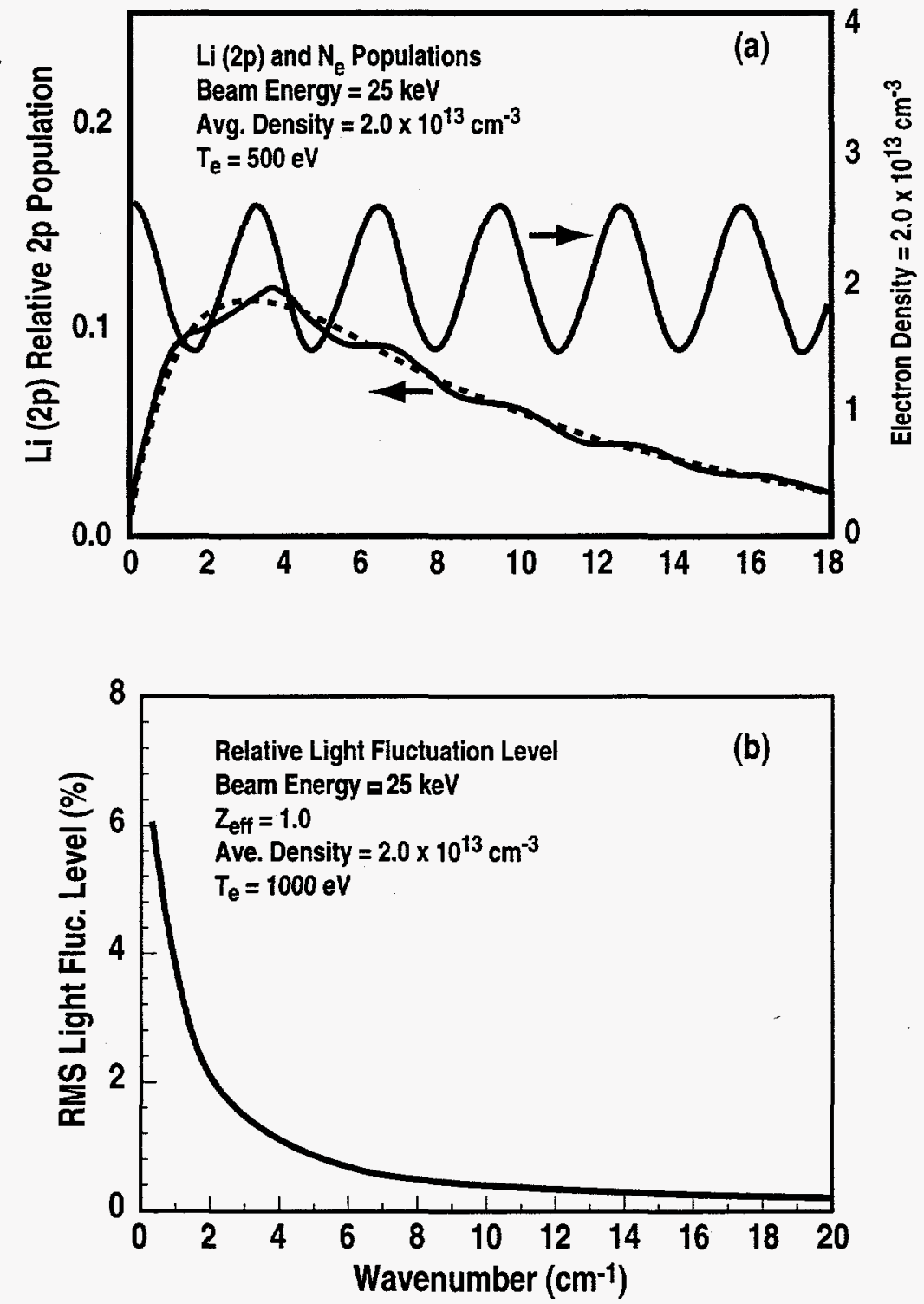

Fig. 7. (a) Sample calculation of the $2 p$ level population as a function of beam penetration. The electron temperature was assumed to be constant $\left(T_{e}=1 \mathrm{keV}\right)$ while the electron density profile was superimposed on it $\left(n_{e}=2 \times 10^{13}, \tilde{n}_{e} / n_{e}=20 \%\right.$ RMS). (b) Relative BES RMS fluctuation level as a function of wavenumber with $\left(\tilde{n}_{e} / n_{e}\right)_{R M S}=0.2$.

\subsection{RECONSTRUCTION OF DENSITY PROFILE}

It will be shown that the underlying electron density profile can be determined by measuring the beam emission over an incomplete section of the emission profile. The chain of detectors need only be calibrated relative to each other, an absolute calibration is not required. This method also has the potential to be extended to the measurement of 
electron density fluctuations, and therefore offers significant advantages over the methods mentioned previously.

Since beam attenuation is proportional to the line integrated density, the effect of any density irregularities tends to be averaged out over the beam path. Therefore a smooth density profile can be used to determine the beam attenuation. The smooth density profile is determined by performing a least squares minimization between the observed light intensity signal $\left(L\left(x_{i}\right)\right)$ and the calculated values for the population of the first excited state. For TEXT the following simple parameterization for the electron density profile was found to provide an good description over a large range of density profiles,

$$
\begin{aligned}
& n_{e}(x)=n_{e o}\left\{\left[1-\left(\frac{r}{a}\right)^{2}\right]^{\gamma}+\delta\right\} \\
& n_{e}(x)=n_{e o} \delta\left(\frac{w-r}{w-a}\right) \\
& (r \leq a),
\end{aligned}
$$

where

$n_{e o}$ is the density at $r=0$, $a$ is the plasma radius, $w$ is the wall radius, $\gamma$ is a profile shape parameter that lies between 0.1 and 2.0, $\delta$ is a parameter that describes the edge density and lies between 0 and 0.1 .

This parameterization of the density profile was found to be flexible enough to accommodate all but the most pathological profiles.

It is now possible to perform a least squares minimization between the experimentally observed BES profile $L\left(x_{i}\right)$ and a series of test profiles $\mathrm{P}_{2}\left(x_{i}, n_{e}, \gamma, \delta\right)$ calculated using Eq. (2).

$$
\chi^{2}=\sum_{i=1}^{n}\left[P_{2}\left(x_{i}, n_{e 0}, \gamma, \delta\right)-\alpha L\left(x_{i}\right)\right]^{2}
$$

The minimum of $\chi^{2}$ is found for various values of $\alpha, n_{e} 0, \alpha, \gamma$, and $\delta$, where $\alpha$ is the scale factor between the experimental and calculated data. By including $\alpha$ as a fitting parameter the difficulty of obtaining an absolute calibration of the PMT chain is avoided. Only a relative channel-to-channel calibration is now necessary. The summation is 
performed over all the " $n$ " data channels. There are various ways of minimizing the $\chi^{2}$ function in Eq. (3), regula falsi, Newton's method, etc. For the present work a network of $1.5 \times 10^{4}$ test profiles $\left[\mathrm{P}_{2}\left(x_{i}, n_{e 0}, \gamma, \delta\right)\right]$ was calculated using the rate equations [Eqs. (1)] and $1.5 \times 10^{4}$ permutation of the parameters $n_{e}, \alpha, \gamma$ and $\delta$. A simple trial and error approach was used to the select the best fit profile.

Once determined by means of least squares minimization the scale factor $\alpha$ need only be recalculated periodically as the beam intensity or the efficiency of the viewing optics changes. By tabulating these profiles the least squares estimates can be calculated very quickly. The speed of this technique allows the large numbers of profiles generated during a plasma discharge to be rapidly analyzed. This speed is particularly important if electron density fluctuations are to be measured. For instance in $\mathrm{H}$-mode plasmas, the fluctuation spectrum may extend up to $500 \mathrm{kHz}$, requiring a digitization rate of $1 \mathrm{MHz}$. If data are acquired for $100 \mathrm{~ms}$, then $10^{5}$ intensity profiles will be generated. Using the methods described Refs. [21-23], each profile would take about two seconds to unfold on a VAX and the entire set of $10^{5}$ profiles would take about 55.6 hours to analyze. This situation is clearly hopeless. Each $\chi^{2}$ evaluation in Eq. (3) takes about $20 \mu$ s on a VAX. Therefore using the methods described above, and assuming about $100 \chi^{2}$ evaluations are necessary to obtain the minimum $\chi^{2}$, gives a total time of $200 \mathrm{~s}$ for the $10^{5}$ profiles.

\subsection{RECONSTRUCTION OF DENSITY FLUCTUATIONS}

The electron density fluctuations can now be calculated by first determining the average density profile and then superimposing the fluctuations on this smooth base profile. Consider the first two coupled rate equations, i.e., consider only the transitions between the ground state, the first excited state and the continuum (ionized) state.

$$
\begin{aligned}
& v_{0} \frac{d P_{1}}{d x}=-\left[k_{12}^{\mathrm{e}(\mathrm{e})}+v_{0} \sigma_{12}^{(\mathrm{e})}+v_{0} \sigma_{1}^{\mathrm{i}(\mathrm{eff})}\right] n_{e} P_{1}+\left[k_{21}^{\mathrm{e}(\mathrm{d})}+v_{0} \sigma_{21}^{\mathrm{i}(\mathrm{d})}\right] n_{e} P_{2}+A_{21} P_{2}, \\
& v_{0} \frac{d P_{2}}{d x}=-\left[k_{12}^{\mathrm{e}(\mathrm{e})}+v_{0} \sigma_{12}^{(\mathrm{i})}\right] n_{e} P_{1}-\left[k_{21}^{\mathrm{e}(\mathrm{d})}+v_{0} \sigma_{21}^{\mathrm{i}(\mathrm{d})}+v_{0} \sigma_{2}^{\mathrm{i}(\mathrm{eff})}\right] n_{e} P_{2}-A_{21} P_{2} .
\end{aligned}
$$

The ionization coefficients $\sigma_{1}^{\mathrm{i}(\text { eff) }}$ and $\sigma_{2}^{\mathrm{i}(\text { eff) }}$ in Eqs. (4) and (5) are effective loss crosssections that include the effects of ion impact ionization/charge exchange and electron impact ionization. The effective ionization cross-section also include ion and electron impact to the excited states principal quantum number $=3,4,5$ because ionization rates are 
much higher from the excited states than from the ground state. Adding Eqs. (4) and (5) gives a simple differential equation for $\mathrm{P}_{1}$.

$$
\frac{d P_{1}}{\mathrm{dx}}+\sigma_{1}^{\mathrm{i}(\mathrm{eff})} n_{e} P_{1}=-\frac{d P_{2}}{d x}-\sigma_{2}^{\mathrm{i}(\mathrm{eff})} n_{e} P_{2}
$$

Equation (6) can be solved for $\mathrm{P}_{1}(x)$ by multiplying by the integrating factor

$$
\lambda(x)=\exp \left[\int \sigma_{1}^{\mathrm{i}(\mathrm{eff})} n_{e} d x\right]
$$

where $n_{e}$ is given by Eq. (2). The right-handside of Eq. (6) is known and the derivative $d \mathrm{P}_{2}\left(x_{i}\right) / d x$ is calculated by making a quadratic fit to the data points $\mathrm{P}_{2}\left(x_{i-1}\right), \mathrm{P}_{2}\left(x_{i}\right)$ and $\mathrm{P}_{2}\left(x_{i+1}\right)$ and then taking the derivative of the fitted quadratic.

Adding fluctuations to $n_{e}(x)$ will not appreciably change either the beam attenuation or the relative populations of the various levels. A simple density profile will suffice to determine $\mathrm{P}_{1}(x)$. Equation (5) can then be used to obtain the density fluctuations $\tilde{n}_{e}(x)$. First, Eq. (5) is linearized $\left(P_{1}=\bar{P}_{1}+\tilde{P}_{1}, P_{2}=\bar{P}_{2}+\tilde{P}_{2}\right.$, and $\left.n_{e}=\bar{n}_{e}+\tilde{n}_{e}\right)$ Then disregarding second order terms and using the fact that

$$
\frac{\tilde{P}_{1}}{\bar{P}_{1}}, \frac{\tilde{P}_{2}}{\bar{P}_{2}} \ll<\frac{\tilde{n}_{e}}{\bar{n}_{e}}
$$

the linearized version of Eq. (5) can be written as

$$
\tilde{n}_{e}\left(x_{\mathrm{i}}\right)=\frac{v_{0} d \tilde{P}_{2}\left(x_{\mathrm{i}}\right) / d x+A_{21} \tilde{P}_{2}\left(x_{\mathrm{i}}\right)}{\left[k_{12}^{\mathrm{e}(\mathrm{e})}+v_{0} \sigma_{12}^{\mathrm{i}(\mathrm{d})}\right] \bar{P}_{1}\left(x_{\mathrm{i}}\right)-\left[\left(k_{21}^{\mathrm{e}(\mathrm{d})}+v_{0} \sigma_{21}^{\mathrm{i}(\mathrm{d})}\right)+v_{0} \sigma_{2}^{\mathrm{i}(\mathrm{eff})}\right] \bar{P}_{2}\left(x_{\mathrm{i}}\right)}
$$

All the terms on the right-hand of Eq. (8) are known. $\bar{P}_{2}$ is known from the least squares fitting procedure. $\bar{P}_{1}$ is known from the fitting procedure or alternatively may be calculated using Eq. (6). As before the derivative $d \tilde{P}_{2}\left(x_{\mathrm{i}}\right) / d x$ is calculated by making a quadratic fit to the data points $\tilde{P}_{2}\left(x_{\mathrm{i}-1}\right), \tilde{P}_{2}\left(x_{\mathrm{i}}\right)$ and $\tilde{P}_{2}\left(x_{\mathrm{i}+1}\right)$ and then taking the derivative of the fitted quadratic. 


\subsection{NUMERICAL EXAMPLE}

To demonstrate the technique the beam emission from a $25 \mathrm{keV}$ beam into a putative TEXT plasma is calculated using the beam attenuation code to give a "measured" $L_{2}\left(x_{i}\right)$ profile which is then unfolded using the above techniques to first determine the smooth electron density profile and thence the electron density fluctuations. In calculating the profile it was assumed that there were 20 detector channels separated by $0.5 \mathrm{~cm}$. The series of data points $L_{2}\left(x_{\mathbf{i}}\right)$ was obtained by first running the forward code to obtain a finely spaced profile of the $2 p$ population $P_{2}(x)$. This finely spaced profile was then divided into 20 segments and an average signal was obtained for each segment.

The results are shown in Fig. 8. The original $\mathrm{n}_{e}(x)$ profile is indicated by the solid line and the unfolded profile is indicated by the dashed line with the solid markers.

The unfolding techniques have proven successful for electron densities less than $4 \times$ $10^{13} \mathrm{~cm}^{-3}$. Beyond that density, the denominator in Eq. (8) approaches zero and the calculated fluctuation levels become unrealistically large. However, the beam attenuation at densities above $5 \times 10^{13} \mathrm{~cm}^{-3}$ was found to be so strong that there was little hope of experimentally measuring light fluctuations in any case, so these somewhat arbitrary corrections were not used. 


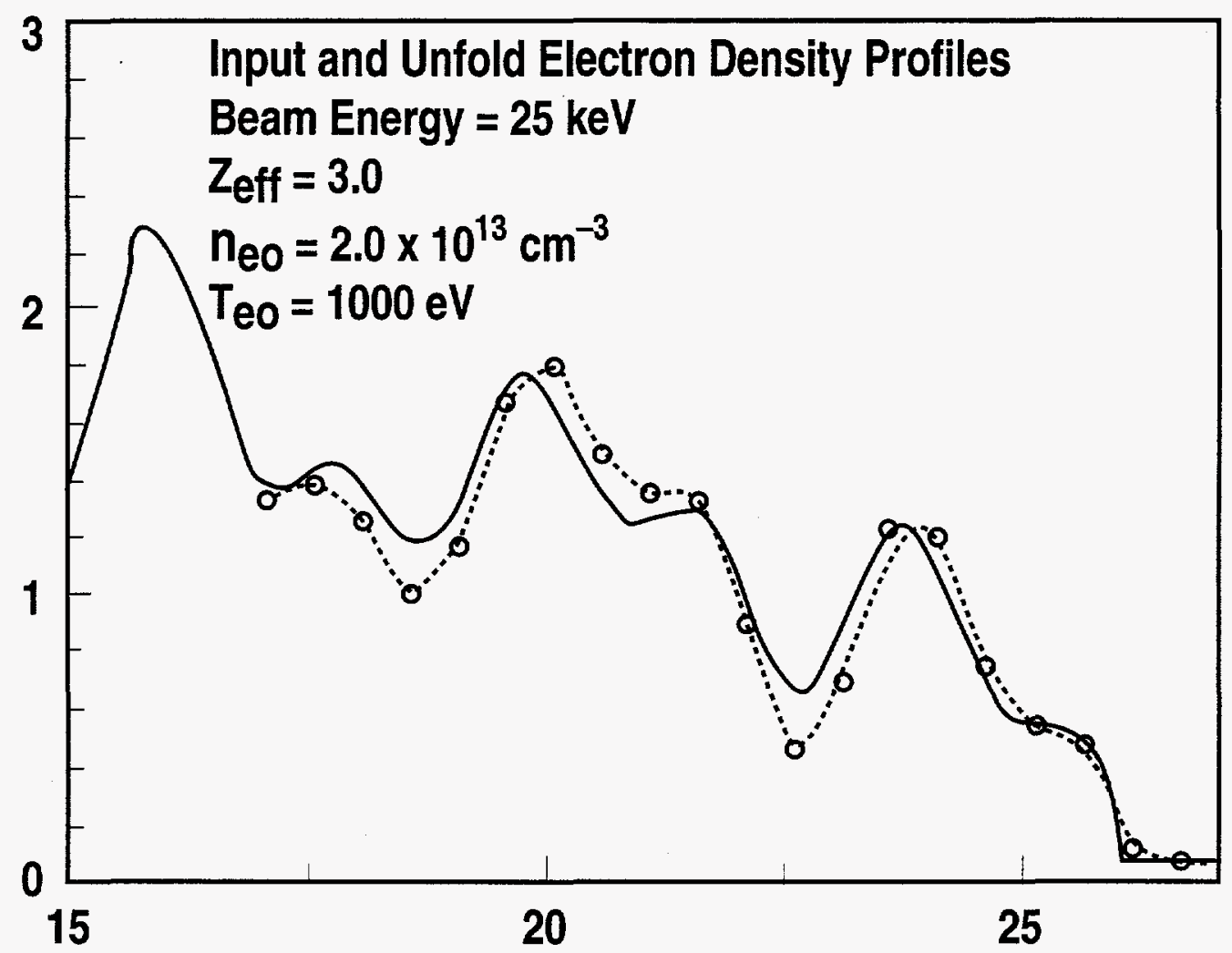

Fig. 8. Numerical example demonstrating unfolding technique. The original profile is shown by the solid line, while the unfolded profile is shown by the dashed line. 


\section{EXPERIMENTAL RESULTS}

Preliminary results have been obtained on the TEXT tokamak and the methods outlined above were used to reconstruct electron density profiles as a function of major radius and of time. The measurement of electron density fluctuations required the accurate measurement of lithium beam emission to better than $1 \%$. The photomultiplier chain was not stable to this degree, so it was not possible to unfold the density fluctuations. An example of the measured fluorescence profile over seven spatial channels separated is shown in Fig. 9(a). The data points are denoted by the solid markers while the least squares fit to these points is denoted by the solid line. The BES signals were obtained by subtracting the plasma background light (obtained during the previous discharge) from the total light signal (plasma background plus beam fluorescence). For this particular plasma discharge (Shot 173987), only seven photo-multiplier tubes were operational. Data were taken using the full 20 channel array described earlier but these have not been analyzed.

Figure 9(b) shows the electron density profile obtained by unfolding the BES signal shown in Fig. 9(a). TEXT had no routine means of measuring the electron density profile, however a semi-empirical formula was found which gave a good fit to the density profile from the core region to the limiter,

$$
\mathrm{n}_{\mathrm{e}}(\mathrm{x})=\mathrm{n}_{\mathrm{e} 0} \exp -\left[\left(\frac{\rho}{\mathrm{w}}\right)^{\mathrm{s}}\right]
$$

Beyond the limiter, the density was assumed to fall off exponentially. The quantities $w$ and $s$ in this formula are functions of the line-averaged density $\left(\bar{n}_{e}\right)$, the plasma current $\left(\mathrm{I}_{\mathrm{p}}\right)$, and the toroidal magnetic field $\left(\mathrm{B}_{\mathrm{t}}\right)$. The solid markers are the densities obtained from the fitting routine. The solid line shows the electron density obtained using Eq. (9) and measured values of $\bar{n}_{e}, I_{p}, B_{t}$. The fit is very good, except around the limiter where the semi-empirical formula (Eq. (9)) is less accurate.

Figure 10 shows the time series of electron density profiles obtained when the fluorescence profiles were unfolded as described above. The time interval between profiles was $25 \mathrm{~ms}$. The plasma density rises for the first $200 \mathrm{~ms}$ and then fluctuations in the profile can be observed. 

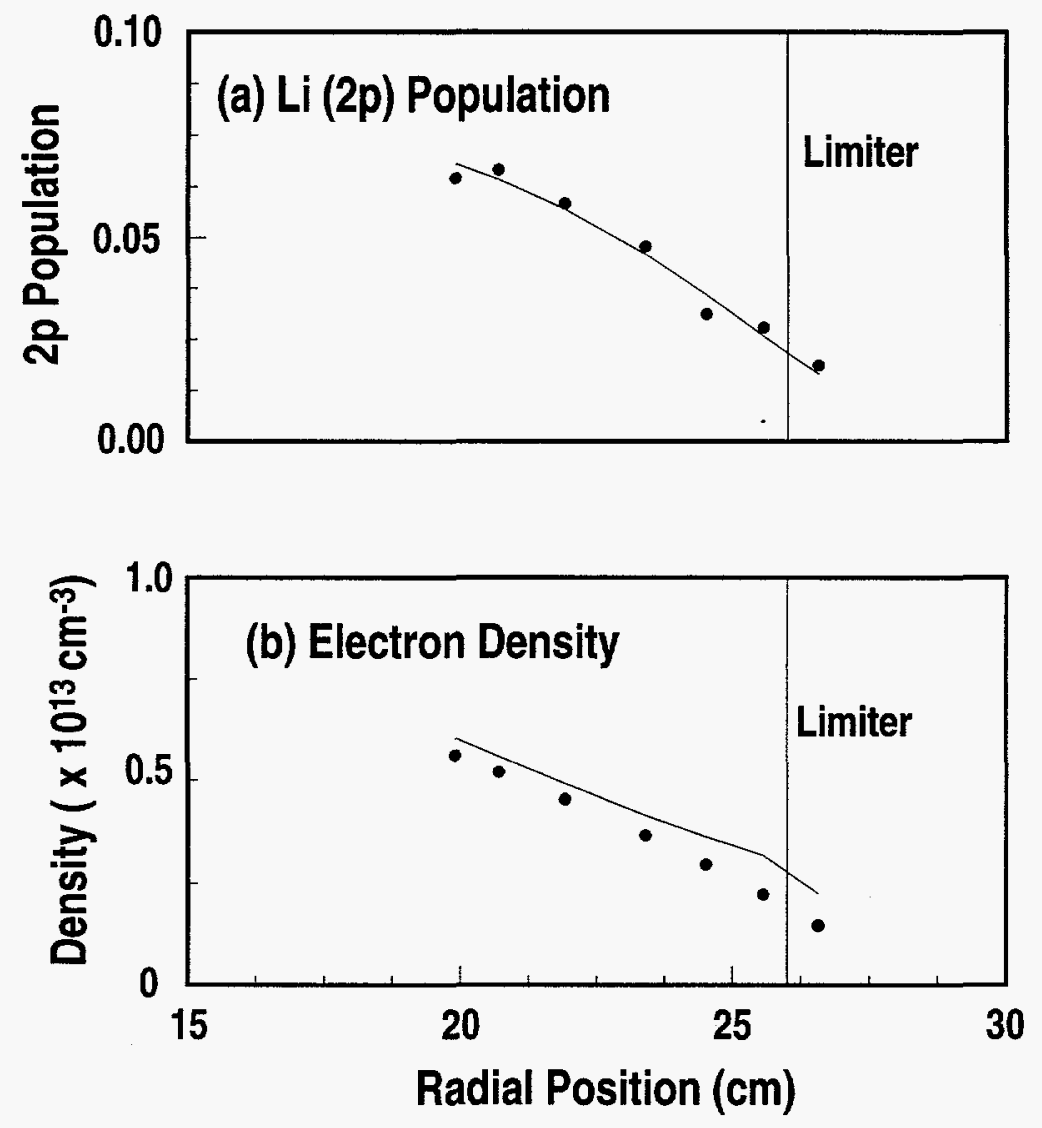

Fig. 9. (a) Example of lithium BES data (solid points) obtained during TEXT discharge 173987. The solid line is the least squares fit to the data. (b) The unfolded electron density profile obtained from the BES profile shown in (a) (solid points) and the density profile obtained using Eq. (9) (solid line). 
Fitted Density Profiles

\section{TEXT-189096}

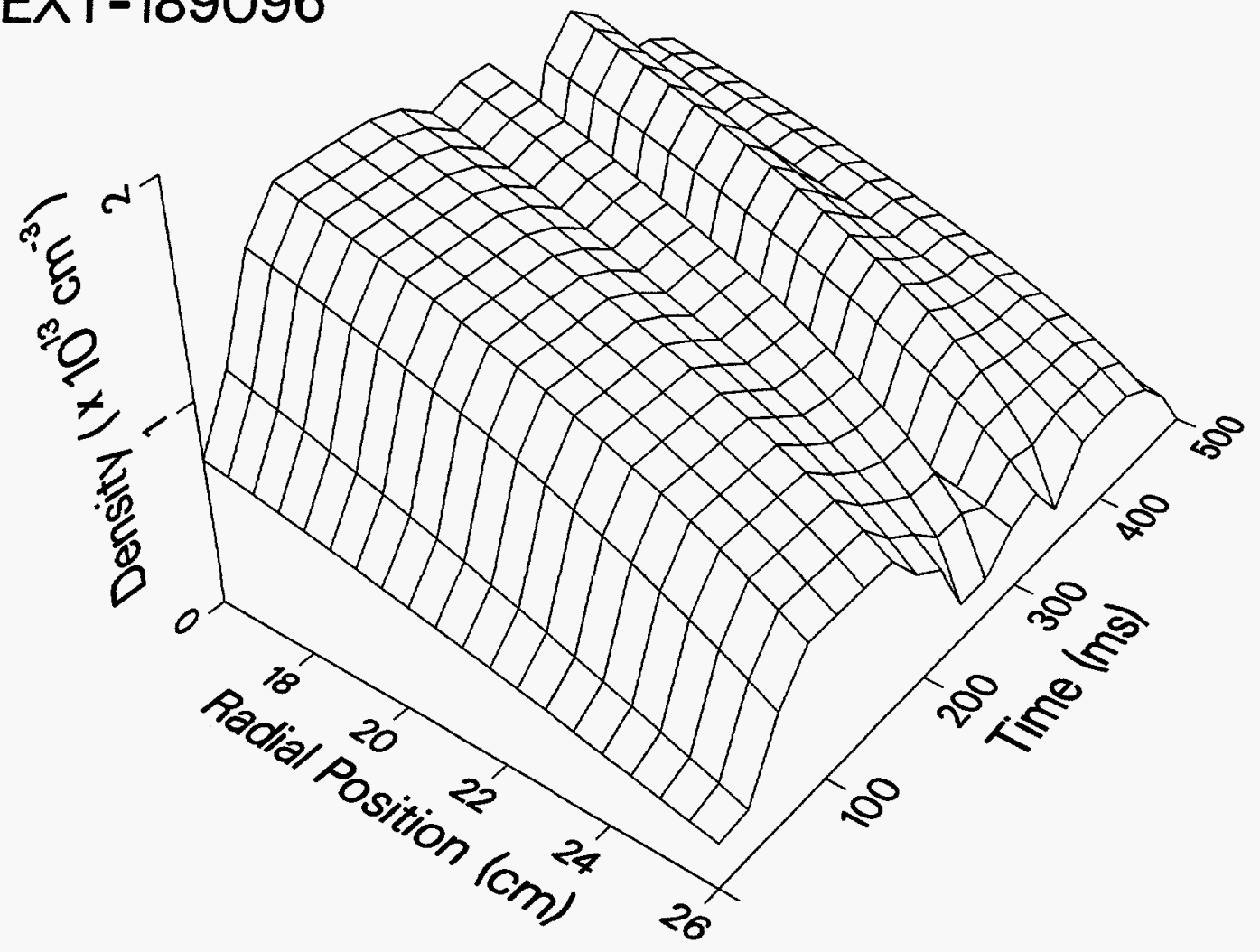

Fig. 10. The time series of reconstructed electron density profiles obtained during TEXT discharge 189096. 


\section{SUMMARY AND FUTURE DIRECTIONS}

Several conclusions can be drawn from our work to date. First, the development and performance of the beam system has enabled us to obtain bright fluorescence signals well inside the plasma for a variety of discharge conditions. The continuous nature of the measurement has allowed us to follow the evolution of the edge plasma for times long compared to the transport timescales. The temporal and spatial resolutions achieved in the data so far have allowed us to address several issues regarding the edge density profile on a truly local scale.

Recent data obtained near the end of TEXT operations appears to have significantly better time resolution and signal-to-noise than the analyzed data shown in the previous section. Improvements in both the temporal and spatial resolution can be addressed through further improvements in detector sensitivity, stability, and configuration. Upgrades to the beam performance and dimensions should extend this capability substantially.

Significant progress has been made in interpretation of the measured fluorescence profile through modeling. Efficient and accurate algorithms have been developed. More analysis development in general is desired to simplify interpreting the data we have already acquired. The capability of making local impurity profile determinations based on lithium-beam doping should also be pursued using suitable spectral filters and view chords. 


\section{REFERENCES}

1. K. H. Burrell, et. al., in 12th Inter. Conf. on Plasma Physics and Controlled Nucl. Fus. Research, Nice, 1988.

2. P. Gohil, et. al, Phys. Rev. Lett. 61, 1603 (1988).

3. K. H. Burrell, Proc 10th Euro. Phys. Soc. Conf. on Controlled Fus. and Plasma Phys, Lisbon, 1993.(GA-A21308).

4. R.G. Groebner, Phys. Fluids B5, 2423 (1993).

5. G.K. McCormick and Z. A. Pietrzyk, J. Nucl. Mater. 162-164, 264 (1989).

6. E. Wolfrum, F. Aumayr, D. Wutte, H.P. Winter, E. Hinze, D. Rusbuldt, and R.P. Schorn, Rev. Sci. Instrum. 64, 2285 (1993).

7. M. Ueda, H. Iguchi, S. Sasaki, and J. Fujita, J. Nucl. Mater. 196Q 198, 923 (1992).

8. W.P. West, D.M. Thomas, E.S. Ensberg, J.S. deGrassie, and J.F. Baur, Rev. Sci. Instrum. 57, 1552 (1986).

9. D.M. Thomas, submitted to Proc. 11th Topical Conference on High Temperature Plasma Diagnostics, Rev. Sci. Instrum., Monterey, California (1996).

10. R.P. Schorn, E. Wolfrum, F. Aumayr, E. Hinze, D. Rusbuldt, and H. Winter, Nucl. Fusion 32, 351 (1992).

11. R.K. Janev, J.J. Smith, F. Aumayr, D. Wutte, M. Schneider, H.P. Winter, and J. Schweinzer, IAEA Rep. No. INDC(NDS)-267 (1993).

12. R.K. Feeney, W.E. Sayle, J.W. Hooper, Rev. Sci. Instrum. 47, 964 (1976).

13. J.P. Blewett, E.J. Jones, Phys. Rev. 50, 464 (1936).

14. A. Septier, H. Leal, Nucl. Instrum. Meth. 29, 527 (1964).

15. O. Heinz and R.T. Reaves, Rev. Sci. Instrum. 39, 1229 (1968).

16. J.R. Pierce, Theory and Design of Electron Beams, 173. (Van Nostrand, 1954). 
17. A. Septier, "Production of Ion Beams of High Intensity," in Septier, A., Focussing of Charged Particles, Vol. 2, 123, (Academic Press, 1967).

18. M. Bacal, et. al., Rev. Sci. Instrum. 45, 769 (1974).

19. E. J. Synakowski, et. al, "Attenuation of a $100 \mathrm{keV}$ Lithium Beam in a Plasma," Proc. ICPEAC Top. Conf. Atom. Proc. Fusion Plasmas, Monterey, 1985.

20. J. M. McChesney, et. al., Bull. Am. Phys. Soc. 36, 2496 (1991), Rev. Sci. Instrum. 63, 4940 (1992).

21. J. Schweinzer, et. al, Plas. Phys. Contr. Fusion 34, 1173 (1992).

22. S. Sasaki, et. al., Rev. Sci. Instrum. 64, 1699 (1993).

23. Z. A. Pietryzk, et. al, Plas. Phys. Contr. Fusion 35, 1725 (1993).

24. An example of this spatial nonuniformity for side-on photomultiplier tubes is given in the Hamamatsu Photomultiplier Tube catalog. 


\section{PUBLICATIONS}

1. W.P. West, J. McChesney, A.M. Howald, M.P. Thomas, and X.Z. Yang, 61, 2929 (1990).

2. J.M. McChesney, W.P. West, and A.M. Howald, Rev. Sci. Instrum. 63, 4940 (1992).

3. J.M. McChesney, "Extracting Electron Density Profiles and Fluctuations from Lithium Beam Fluorescence Profiles," GA-D21569, February 1994.

4. A.M. Howald, J.M. McChesney, and W.P. West, Rev. Sci. Instrum. 66, 312 (1995). 